\title{
Study about Hydrophilic Modification of PTFE Membrane
}

\author{
Yu-Hui Zeng ${ }^{\mathrm{a}}$, Ting-Ting Zhang ${ }^{\mathrm{a}}$, Yi-Ren Chen ${ }^{\mathrm{a}, \mathrm{b}, *}$ \\ ${ }^{a}$ School of Textile Science and Engineering, Wuhan Textile University, Wuhan 430200, China \\ ${ }^{\mathrm{b}}$ Textile Fiber and Products Ministry of Education Key Laboratory
}

\begin{abstract}
Polytetrafluoroethylene (PTFE) membrane is widely used in medicine, clothing, chemical, electronics and other fields. However, due to the highly symmetrical structure of the PTFE, the absence of reactive radical, high crystallinity, low surface energy and poor surface wetting, these properties of PTFE affect its compound with other materials. In order to improve the adhesion property of PTEF membrane, the surface must be modified. In this paper, the surface melting deposition method was used to improve the hydrophilicity of PTFE membrane. The PTFE membrane is immersed in a suspension of nano- $\mathrm{TiO}_{2}$, and then deposited by melting at a high temperature. Sintering and depositing $\mathrm{TiO}_{2}$ particles on the surface of the PTFE membrane. The molecules of the viscous substance enter the surface layer of the PTFE membrane to form a modified surface layer. The interface strength of PTFE membrane is increased, and the surface activity of PTEF membrane is increased, and the hydrophilicity of the PTFE membrane is improved. It is easier that PTFE membrane is combined with other materials. The best treatment process for the modification by melting deposition is as follows: the optimum treatment temperature is $350{ }^{\circ} \mathrm{C}$, and the optimum treatment time is $30 \mathrm{~min}$.
\end{abstract}

Keywords: PTFE Membrane; Surface Modification; Surface Melting Deposition Method; Contact Angle Infrared

\section{Introduction}

In the late 1970s, with the advent of the second-generation Gore-Tex membrane, a large number of PTFE membrane products were developed and put on the market. Waterproof and moisture permeable fabric is one of its important applications. This waterproof and moisture permeable laminate fabric is formed by laminating a PTFE microporous membrane with a common fabric. The laminated fabrics are widely used in medicine, hygiene, sportswear, and other areas. Compared with high-density fabrics and coated fabrics, it has obvious technical advantages [1]. However, the extremely low surface activity and poor adhesion property of the PTFE membrane limit its compounding with other materials and often cause peeling off between the membrane and the fabric after washing several times, which affects the appearance and durability.

\footnotetext{
${ }^{*}$ Corresponding author.

Email address: yiren.chen@wtu.edu.cn (Yi-Ren Chen).
} 
The adhesion property of the PTFE membrane is poor, due to wetting ability of PTFE membrane is not good. The contact angle of PTFE membrane to water is relatively large, which can reach more than $110^{\circ}[2]$. PTFE is a high crystalline material and therefore it is chemically stable. Its swelling and dissolution are more difficult than the amorphous polymers. When forming bonding with solvent-based adhesives, it is difficult for a polymer chain to chain intermolecular domain diffusion and entanglement where it is unable to form a strong adhesion bond [3]. Because PTFE membrane structure is highly symmetrical and it does not contain active groups, contains high crystallinity, has low surface energy as well as very poor wettability, it is difficult for PTFE membrane to interfusion with other materials, and so it is difficult to produce tight adhesion with other materials [4]. In order to improve its adhesion performance, it is advantageous to compound it with other materials. There are various methods to change the surface structure of PTFE membrane. In order to achieve the purpose of surface modification, the current treatment methods can be divided into two categories; one is chemical method: surface chemical grafting and superficial treatment; the other is physical methods: including mechanical coating method, flame method, Plasma Jet method, Corona treatment method [5].

In this paper, surface deposition and fusion method is used to treat the surface of PTFE membrane. The $\mathrm{TiO}_{2}$ particles are deposited on the surface of PTFE membrane, forming a layer of modified layer which is embedded with adhesive. As the molecule of the adhesive enters the surface molecule, the interface strength increases, the wettability increases, the surface activity improves, and it becomes easy to be compounded with other materials [6].

\section{Experimental}

\subsection{Experimental Materials}

PTFE microporous membrane, Xinxiang Huayuan God-Care Co., Ltd. production. Tetrafluoroethylene (PTFE) microporous film is a lightweight, flexible film that is obtained using a special biaxial stretching process. There are about 9 billion micropores per square inch, 20 times the pore size and 20 thousand times smaller than the water droplet. It has excellent waterproof and windproof property along with great moisture permeability, thermal insulation and corrosion resistance. It is an ideal material for making advanced composite fabrics. At the same time, its water pressure resistance and special micro porosity are also widely used in the field of membrane permeation, such as construction and water treatment. $\mathrm{TiO}_{2}$ powder, manufactured by Degussa company; PEG6000, chemically pure; PTFE emulsion; NaOH, analytical grade/analytically pure, Wuhan Yatai Chemical Reagent Co., Ltd.; Acetone, industrial use.

\subsection{Experimental Equipment}

Ultrasonic cleaning machine, TEA/1006, Shenzhen Times/era Ultrasound Equipment Co., Ltd. production; Marv/MUFU Furnace, Wuhan element technology production; Contact angle measuring instrument, Shanghai JC2000A static drop contact angle measuring instrument; Infrared spectrometer, TENSOR27, Germany Bruker spectrometer company; X-ray diffractometer, Ultima3 type, manufactured by Rigaku Corporation. 


\subsection{Experimental Steps}

Membrane cleaning: The PTFE film is put into the industrial acetone, and the ultrasonic wave is used to oscillate a certain time to remove the impurities on the surface.

1) Preparation of suspension: At first $1.0 \mathrm{~g}$ of $\mathrm{TiO}_{2}$ powder was taken into a beaker, and then $20 \mathrm{~g}$ of distilled water was added; then PEG was added in $0.3 \%$ of the mass ratio as a dispersant; $\mathrm{NaOH}$ was added to adjust the $\mathrm{pH}$ value of 9-11. And then the beaker was placed into the ultrasonic cleaning Machine oscillation for 15 minutes. Thus, was obtained the required $5 \% \mathrm{TiO}_{2}$ suspension.

2) The proper amount of PTFE emulsion is added in the $\mathrm{TiO}_{2}$ suspension.

3) The cleaned PTFE film is dried and put into the solution 5min in the step 2.3. (3).

4) The PTFE film out of the solution, put it in the oven and bake in the Marv furnace.

\subsection{Test}

\subsubsection{Contact Angle Test of PTFE Film}

The static contact angle between sample and water was determined by Shanghai JC2000A drop contact angle measuring instrument (shown in Fig. 1). In this experiment, the liquid droplet method is used to determine the contact angle of water. The method is that the sample to be measured is placed on a thermostatic platform, the platform is adjustable, and the droplets are dripped on the surface of the sample so that they naturally form droplets in a state of equilibrium until the solid-liquid gas three-phase boundary reaches equilibrium. Angle method to measure the size of the contact angle can be directly read.

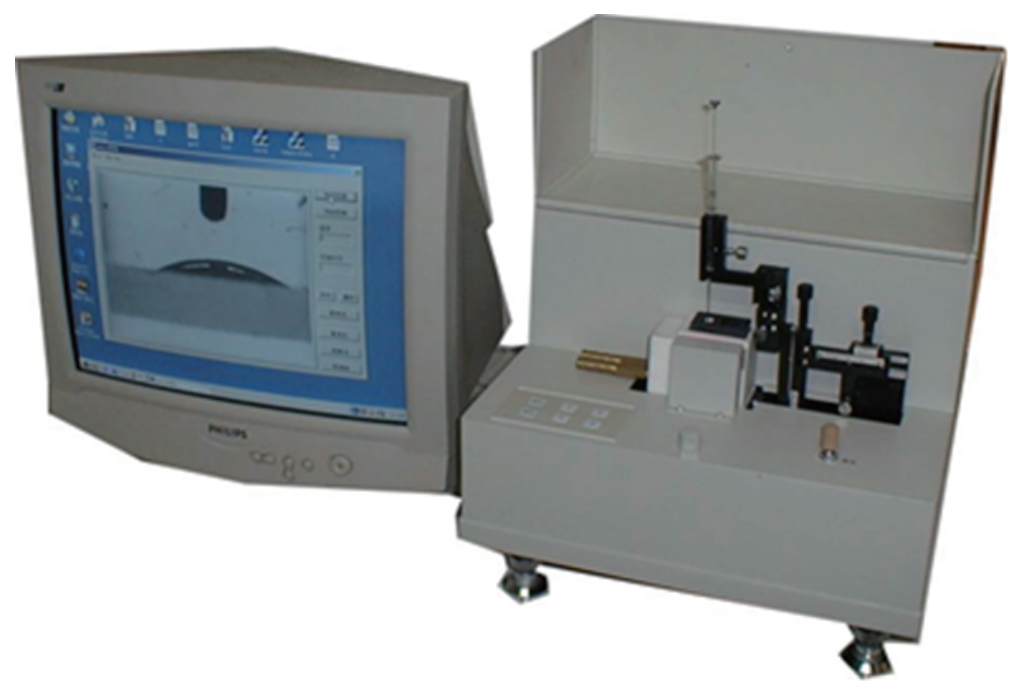

Fig. 1: JC2000A drop contact angle measuring instrument

\subsubsection{Infrared Spectrum of PTFE Membrane}

The TENSOR27 infrared spectrometer was used to test the PTFE membrane before and after the modification, and to observe whether a new absorption peak appeared in the Atlas, that 
is, whether a new group was generated. Without the appearance of a new absorption peak, it is possible to verify that the hydrophilicity of the PTFE membrane is improved because the morphology of the PTFE membrane surface is changed at high temperature, and some $\mathrm{TiO}_{2}$ with high surface energy and easy adhesion is embedded. Thus, can be said that the surface deposition melting method modification is effective.

\subsubsection{X-ray Diffraction Analysis of PTFE Membrane}

Through the analysis of the X diffraction pattern, it is observed whether the crystallinity decreases with the increase of the sintering temperature. If the crystallinity is reduced, it obviously indicates that the crystallization morphology of PTFE membrane surface changes at high temperature, and embeds some $\mathrm{TiO}_{2}$ with high and easy adhesion to the surface, so that the hydrophilicity of PTFE membrane is improved.

\section{Results and Analysis}

\subsection{Contact Angle Test Results and Analysis}

The sintering time of $10 \mathrm{~min}, 20 \mathrm{~min}, 30 \mathrm{~min}$ and $40 \mathrm{~min}$ with the sintering temperature of $300{ }^{\circ} \mathrm{C}$, $350{ }^{\circ} \mathrm{C}, 380{ }^{\circ} \mathrm{C}$ and $400{ }^{\circ} \mathrm{C}$ were respectively used. After the PTFE membrane impregnated with $\mathrm{TiO}_{2}$ emulsion was melted and deposited, the contact angle of the PTFE membrane on water was measured. Take the average values of the contact angle at 8 positions. The results are shown in Table 1 and Fig. 2.

Table 1: Contact angle of PTFE membrane to water on different process conditions

\begin{tabular}{ccccc}
\hline \multirow{2}{*}{ Temperature } & \multicolumn{4}{c}{ Contact Angle $\left(^{\circ}\right)$} \\
\cline { 2 - 5 } & 10 min & 20 min & 30 min & 40 min \\
\hline $300{ }^{\circ} \mathrm{C}$ & 116 & 112 & 105 & 99 \\
$350{ }^{\circ} \mathrm{C}$ & 98 & 95 & 79 & 80 \\
$380^{\circ} \mathrm{C}$ & 96 & 80 & 78 & 80 \\
$400^{\circ} \mathrm{C}$ & 96 & 79 & 75 & 78 \\
\hline
\end{tabular}

Note: The contact angles (8-times averages) of the unmodified PTFE membrane were $122^{\circ}$

From Table 1 and Fig. 2, it can be seen that the contact angle of PTFE membrane decreases with the increase of sintering time, and the contact angle of PTFE membrane reaches a smaller value after sintering for $30 \mathrm{~min}$. So, we can conclude it as the best sintering time. When the time is $30 \mathrm{~min}$ and the temperature is $300{ }^{\circ} \mathrm{C}$ the contact angle is $105^{\circ}$; when the temperature rises to $350{ }^{\circ} \mathrm{C}$, its contact angle quickly reduces to the $79^{\circ}$ and when the temperature is $380{ }^{\circ} \mathrm{C}$ and $400{ }^{\circ} \mathrm{C}$, the contact angle is $78^{\circ}$ and $75^{\circ}$ respectively. The contact angle changes a little when the temperature continues to rise. At the same time, the surface shape of the membrane deforms with the increase of temperature. We conclude that $350{ }^{\circ} \mathrm{C}$ is the best sintering temperature.

The contact angle of the untreated PTFE membrane is shown in Fig. 3, and the contact angle of the PTFE membrane modified by the nano- $\mathrm{TiO}_{2}$ melting deposition method is shown in Fig. 4. 


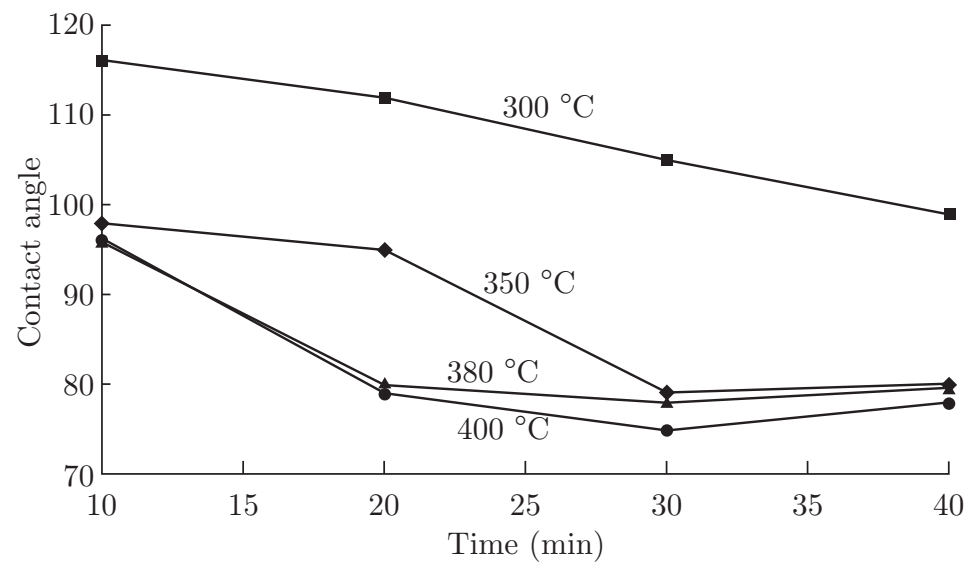

Fig. 2: Contact angle of PTFE membrane to water on different process conditions

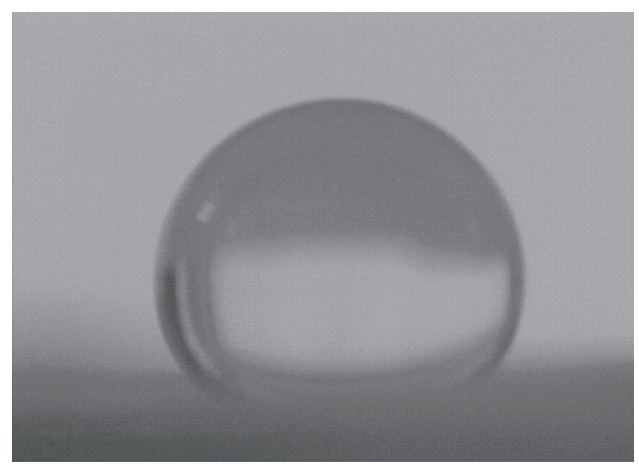

Fig. 3: PTFE membrane contact angle before treatment

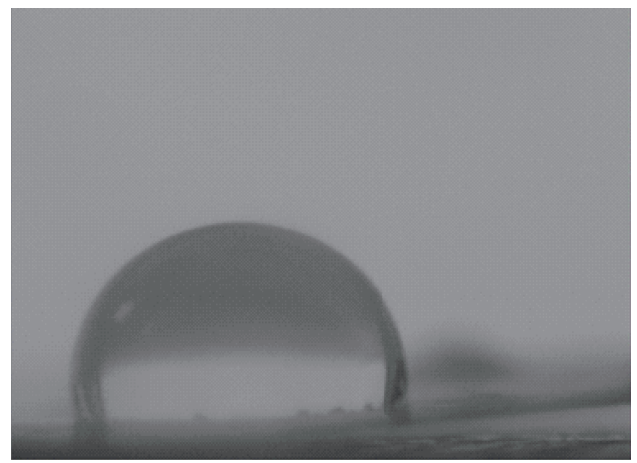

Fig. 4: PTFE membrane contact angle after treatment

\subsection{Infrared Spectroscopy}

The infrared spectra of the untreated PTFE membrane and the PTFE membrane under different treatment conditions is shown in Fig. 5.

As shown in Fig. 5, no new absorption peak, that is, no new group generation is found on the PTFE membrane surface no matter how the sintering time and the sintering temperature change. This shows the improvement of the hydrophilicity of the PTFE membrane due to the change in the crystalline structure of the PTFE membrane surface at high temperature, which has some $\mathrm{TiO}_{2}$ with high surface energy and easy adhesion embedded in the surface. 


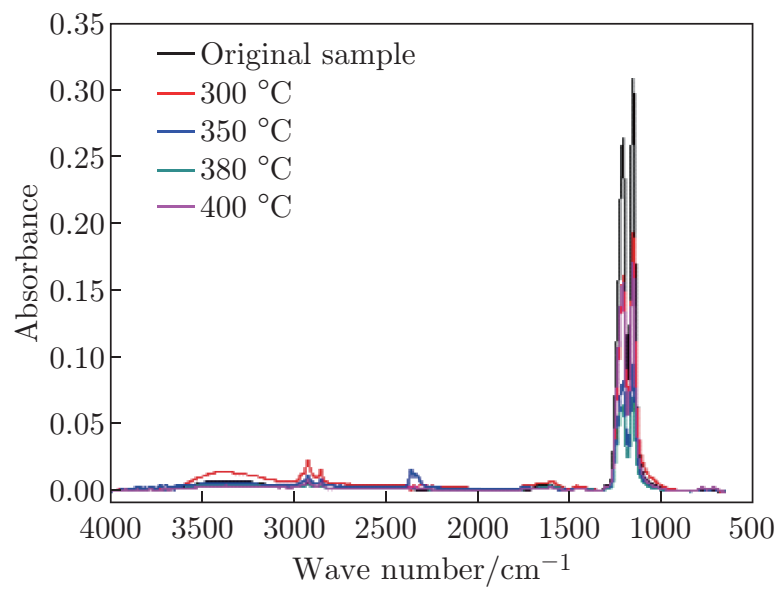

Fig. 5: Infrared spectra of the modified PTFE membrane before and after modification

\subsection{X-ray Diffraction Pattern Analysis}

X-ray diffraction patterns of PTFE membrane before and after treatment modified by sintering at $300{ }^{\circ} \mathrm{C}, 350{ }^{\circ} \mathrm{C}, 380{ }^{\circ} \mathrm{C}$, and $400{ }^{\circ} \mathrm{C}$ can be shown in Fig. 6-Fig. 10 .

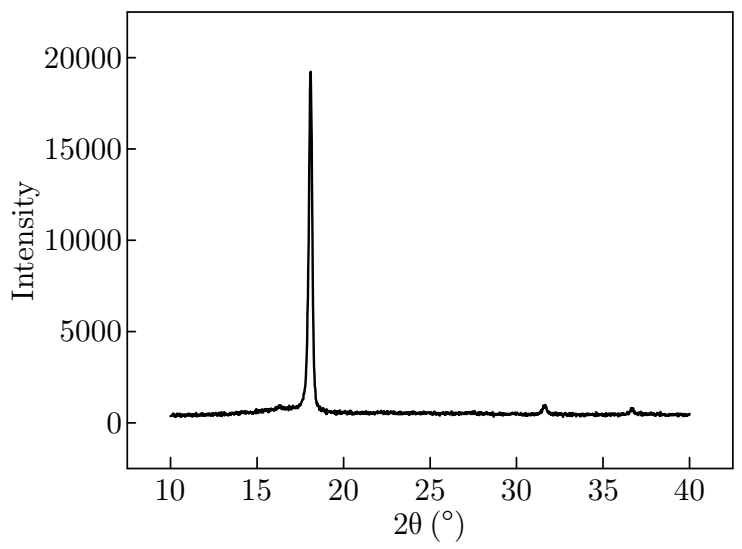

Fig. 6: X-ray diffraction pattern of PTFE membrane before treatment

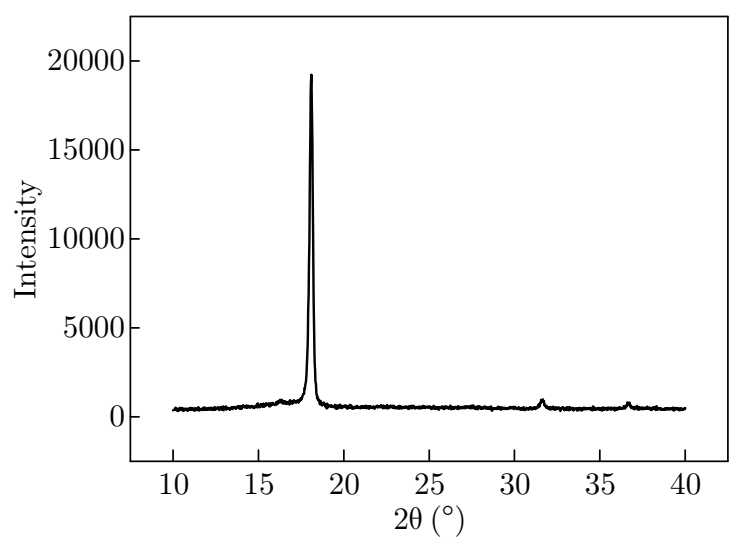

Fig. 7: X-ray diffraction pattern of PTFE membrane sintered at $300{ }^{\circ} \mathrm{C}$ 


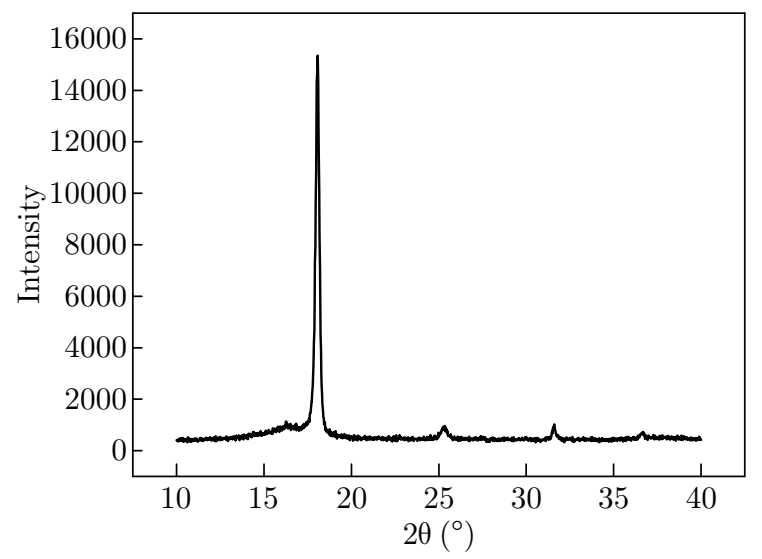

Fig. 8: X-ray diffraction of PTFE membrane after sintering at high temperature $350{ }^{\circ} \mathrm{C}$

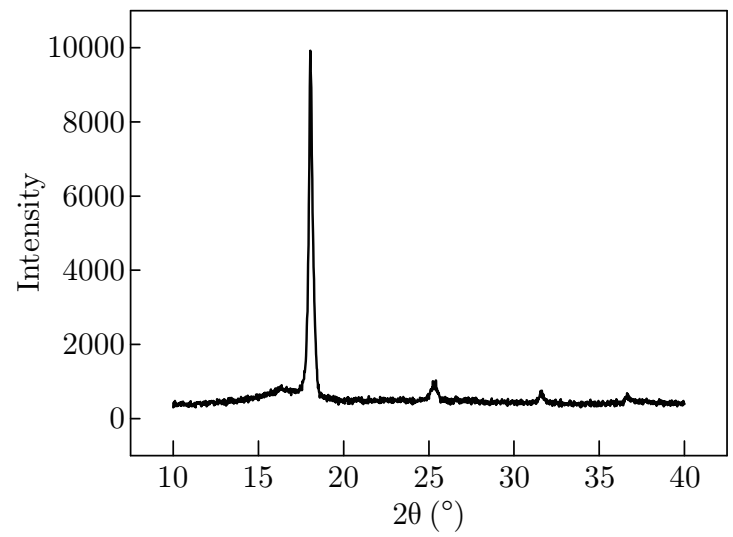

Fig. 9: $380{ }^{\circ} \mathrm{C}$ X-ray diffraction of PTFE membrane after sintering at high temperature

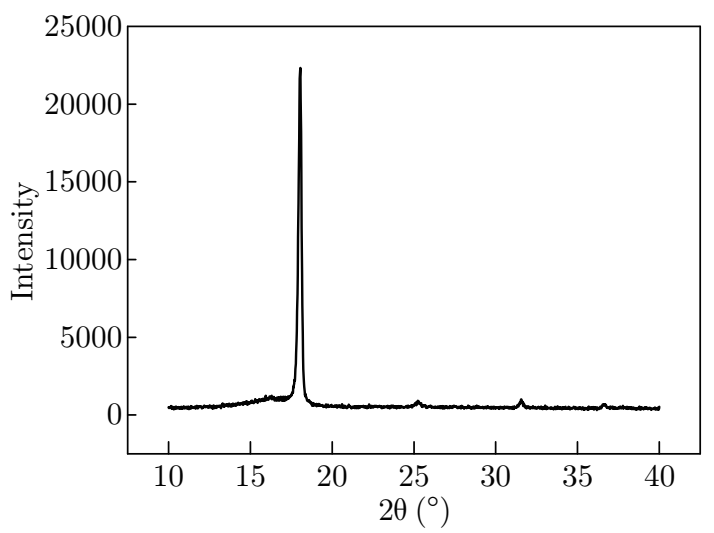

Fig. 10: $400{ }^{\circ} \mathrm{C}$ X-ray diffraction of PTFE membrane after sintering at high temperature

Fig. 6 to Fig. 10 shows the X-ray diffraction of the PTFE membrane prior to processing and $300{ }^{\circ} \mathrm{C}, 350{ }^{\circ} \mathrm{C}, 380{ }^{\circ} \mathrm{C}$ and $400{ }^{\circ} \mathrm{C}$ sintering. Through analysis and calculation, it was shown that with the increase of sintering temperature, the crystallinity of the PTFE membrane is reduced obviously. The original crystallinity of the PTFE membrane is $80 \%$, and the crystallinity of PTFE membrane is $72 \%$ after $400{ }^{\circ} \mathrm{C}$ sintering. After high temperature treatment, the surface crystalline morphology of PTFE membrane changed. 


\section{Conclusion}

4.1 With the increase of sintering time, the contact angle is gradually reduced. At 30 minutes of sintering, the contact angle reaches a small value. The influence on the contact angle is not obvious when the sintering time continues to increase. If the temperature is too high, the shape of the PTFE microporous membrane changes, and at the same time, some micropores are blocked. The research shows that the best treatment process is: the optimal processing temperature is $350{ }^{\circ} \mathrm{C}$, and the optimal processing time is $30 \mathrm{~min}$.

4.2 By X-ray diffraction analysis shown that the crystallinity of the PTFE membrane decreases with the increase of the sintering temperature. It was found by infrared spectroscopy that no new absorption peak was observed. It means no new group generation on the PTFE membrane surface. The hydrophilicity of the PTFE membrane is improved because the crystal morphology of the PTFE membrane surface changes at high temperatures, and some $\mathrm{TiO}_{2}$ with high surface energy and easy adhesion are embedded.

4.3 It is feasible to use $\mathrm{TiO}_{2}$ to melt deposition on the surface of PTFE membrane to improve the adhesion property of PTFE membrane.

\section{References}

[1] Shen YF, Huang BX, Huang L. Manufacturing Technology and Application of PTFE Microporous Membrane. Shandong Chemical Industry: 2012, (12): 51-53.

[2] Sun HX, Zhang L, Chen HL. Advances on hydrophilic modification of polytetrafluoroethylene porous membrane. CHEMICAL INDUSTRY AND ENGINEERING PROGRESS: 2006, (4): 378382.

[3] Fu NQ, Wang YY, Meng JY, Sun X, Chen FF. Effects of Cold Plasma Treatment Process on Surface Properties of Acrylonitrile-butadiene Rubber. Surface Technology: 2016, 45(12): 117-123.

[4] Wang YY, Meng JY, Wang YP. Effect on PTFE Surface Property Treated by Low Temperature Plasma. Journal of Aeronautical Materials: 2009, (5): 77-81.

[5] Li P, Fan LY, Fei CJ. Study on the preparation method of hydrophilic polytetrafluoroethylene microporous membrane. New Chemical Materials: 2016, 44(03): 62-63+69.

[6] Zhang TT, Wu SF, Hasan MR, Chen YR. Study on the Modification of PTFE Film Surface by Deposition and Melting Method. The 11th Textile Bioengineering and Informatics Symposium Proceedings: 2018: 1041-1047. 